\title{
Editorial
}

\section{Advances in Computational Fluid Dynamics}

\author{
Mahesh T. Dhotre, ${ }^{1}$ Nandkishor Krishnarao Nere, ${ }^{2}$ Sreepriya Vedantam, ${ }^{3}$ and Mandar Tabib ${ }^{4}$ \\ ${ }^{1}$ ABB Switzerland Ltd., Fabrikstrasse 13a, 5400 Baden, Switzerland \\ ${ }^{2}$ Aditya Birla Science and Technology Company Ltd., Plot No. 1 \& 1-A/1, MIDC Taloja, Panvel, Maharashtra 410208, India \\ ${ }^{3}$ CEPD Division, National Chemical Laboratory (NCL), Pashan Road, Pune 411008, India \\ ${ }^{4}$ Flow Technology Group, SINTEF Materials and Chemistry, Grønbrygge, Gløshaugen, Trondheim, Norway
}

Correspondence should be addressed to Mahesh T. Dhotre; mahesh.dhotre@ch.abb.com

Received 19 December 2012; Accepted 19 December 2012

Copyright (C) 2013 Mahesh T. Dhotre et al. This is an open access article distributed under the Creative Commons Attribution License, which permits unrestricted use, distribution, and reproduction in any medium, provided the original work is properly cited.

\section{Introduction}

Over the past few decades, Computational Fluid Dynamics (CFD) has emerged as a powerful tool for the design and optimization of new products and processes. It is widely used in a variety of applications and industries such as chemical, petroleum, aerospace, automotive, power generation, polymer processing, medical research, construction, meteorology, and so forth. Some of the key offerings include the design reliability and reduction in the time and cost of the product development.

A significant research is being carried out in a quest to model the physics accurately and to develop robust and efficient numerics (solution algorithms and accurate discretization methods). The interaction between the CFD and disciplines like solid mechanics and optimization algorithm is also evolving. However, focus of the research still remains on the most challenging topics in fluid mechanics, namely, the turbulence and the multiphase flow that also form the common theme of papers presented in this special issue.

1.1. Turbulence. Direct Numerical Simulation approach requires no modeling. However, it remains a research tool with today's available computer power and useful for gaining insight into turbulence physics, calibrating the models, and scaling laws. For practical engineering applications, a great deal of efforts are devoted to the development of turbulence models (e.g., approaches based on Reynolds Averaged Navier Stokes (RANS) equations). The strengths and weaknesses of these models are now well known and alternatives approaches such as Large Eddy Simulation (LES) and hybrid RANS/LES turbulence models are being explored.

At this stage, the development in high-performance computing has huge impact on the computational progress. The RANS approach is being used for more complex problems involving unsteadiness, coupled physics, and complex geometry. LES has become feasible for the simplified industrial systems and even being attempted for more realistic geometries. However, bottleneck is better models for small scale turbulence and associated computational cost for integrating CFD with the design cycle in the industry.

1.2. Multiphase Flow. Multiphase flow combined with the turbulence pose formidable challenge for simulation even for the dilute dispersed phase. Many approaches are being considered mainly through, variation in reference frame representations (Eulerian or Lagrangian); phase coupling (intraphase and/or interphase coupling); and particle/flow details (e.g., resolution around single bubble or bulk description of many bubbles). These approaches come with huge variations in computational time and predictive capability.

The challenge is also to incorporate important flow details such as (a) the impact of deformation rate on bubble/particle breakup and coalescence, (b) the phenomenon like turbulent modulation (dispersed phase particle can either augment or attenuate the turbulence in the continuous phase), (c) turbulent dispersion (the eddies in the continuous phase can cause dispersed particles to be transported from regions of higher concentration to regions of lower concentration). 
The multiphase research is being driven by all these aforementioned challenges and progress is under way. The significant contributions are required to arrive to a stage where it can be used with a higher degree of confidence.

\section{Summary}

The special issue covers a broad spectrum of CFD applications and developments. There are sixteen papers presented; two reviews discuss the state-of-the-art CFD modeling and its applications for the dispersed bubbly flows and annular flow in centrifugal extractors. Three papers focus on important multiphase flows topics: the bubble dynamics in the boiling phenomena, bubble coalescence and breakup in gas liquid bubbly flows and effect of drag models and turbulent dispersion in solid suspension. CFD and design related applications have been explored in papers for twoopposed-jet microextractor, high voltage circuit breaker and corrugated sheets of packing in a gas liquid contactors. Optimization algorithm and CFD coupling is investigated in one paper for the design of wind turbine airfoil. CFD analysis is presented in two papers for predicting the heat release rate in fire application and tip structure design of LVAD inflow cannula in medical application. One paper examines the influence of gravity on countercurrent imbibition of twophase flow in porous media. LES approach is explored in two papers for applications in combustion and mixing in stirred precipitator. In another two separate papers, simulations are presented investigating chemical kinetics of nitrogen oxides formation and flow pattern in submerged channels at electrodes.

We hope that the issue will be interesting for scientists from both the industry and academia as well as for practicing engineers involved in CFD simulations. The earnest desire of this issue is also to provide insights into some of the open questions by throwing light on the requirements for further research.

Mahesh T. Dhotre

Nandkishor Nere

Sreepriya Vedantam

Mandar Tabib 

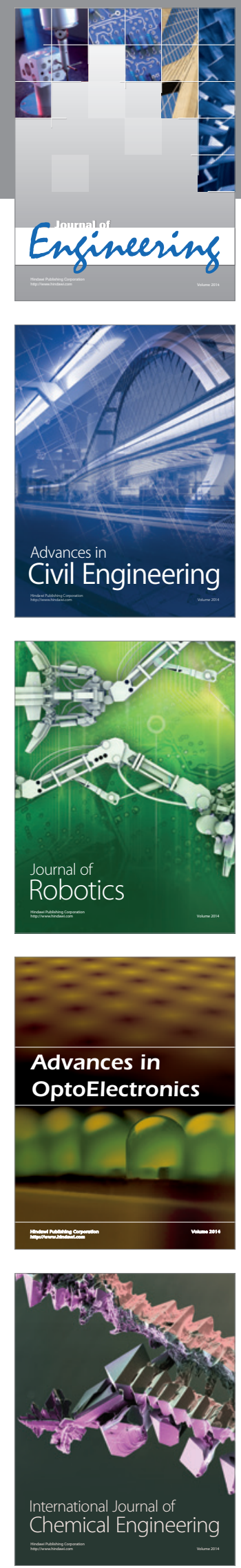

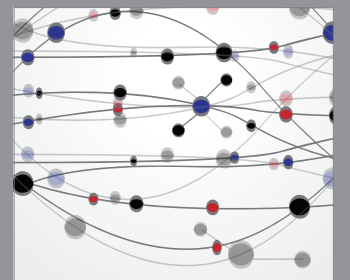

The Scientific World Journal
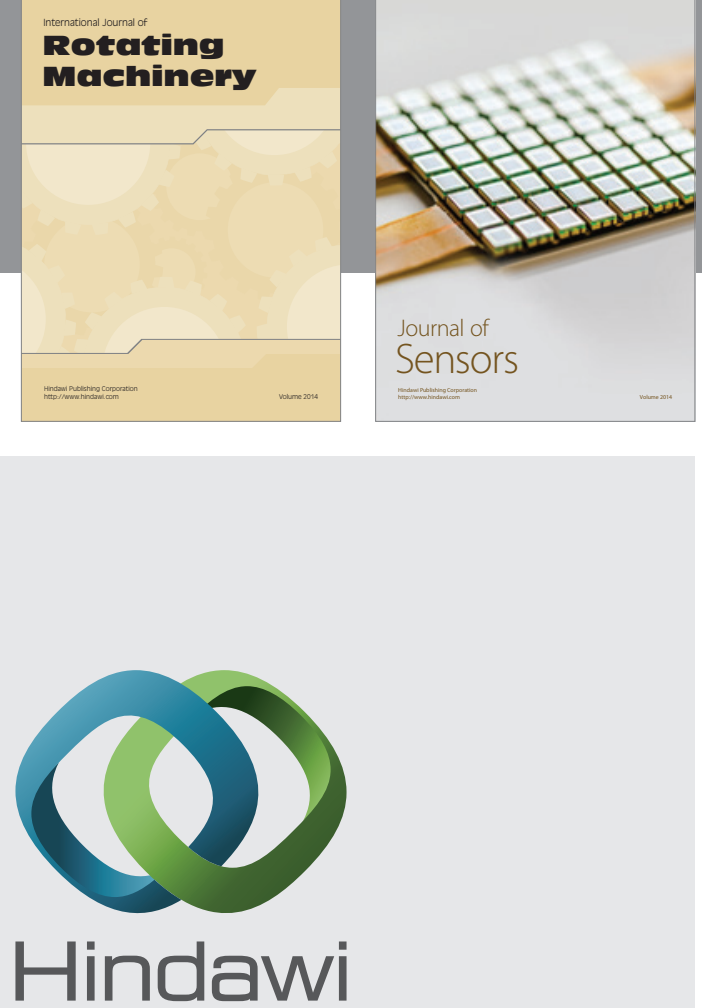

Submit your manuscripts at http://www.hindawi.com
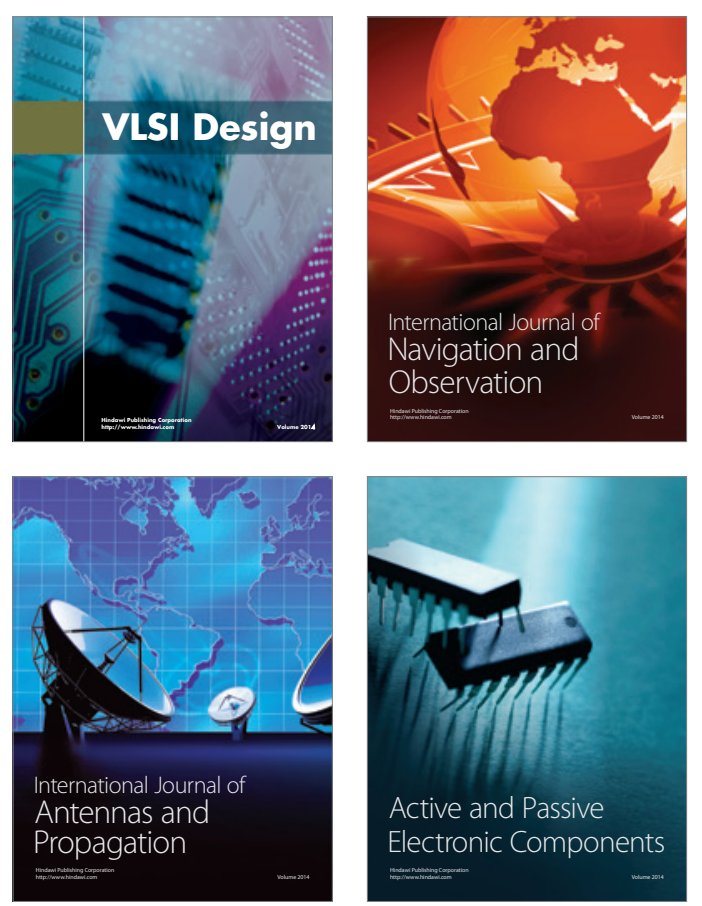
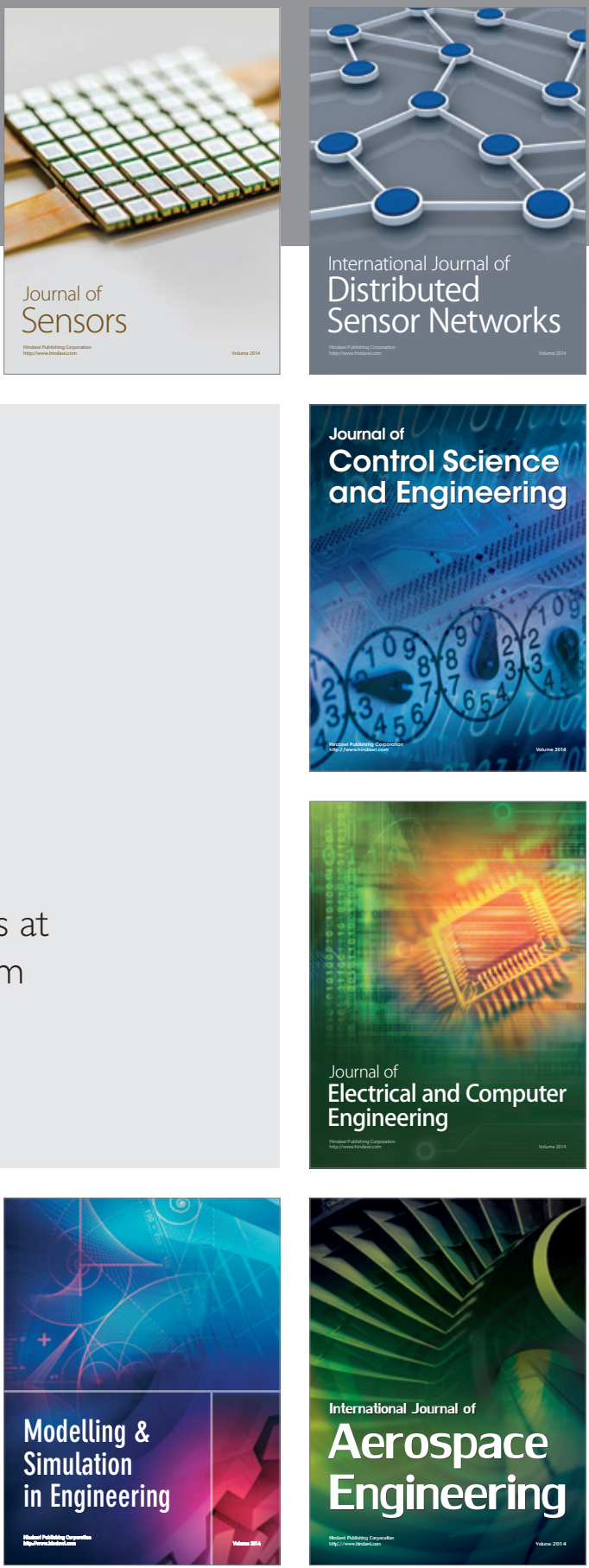

Journal of

Control Science

and Engineering
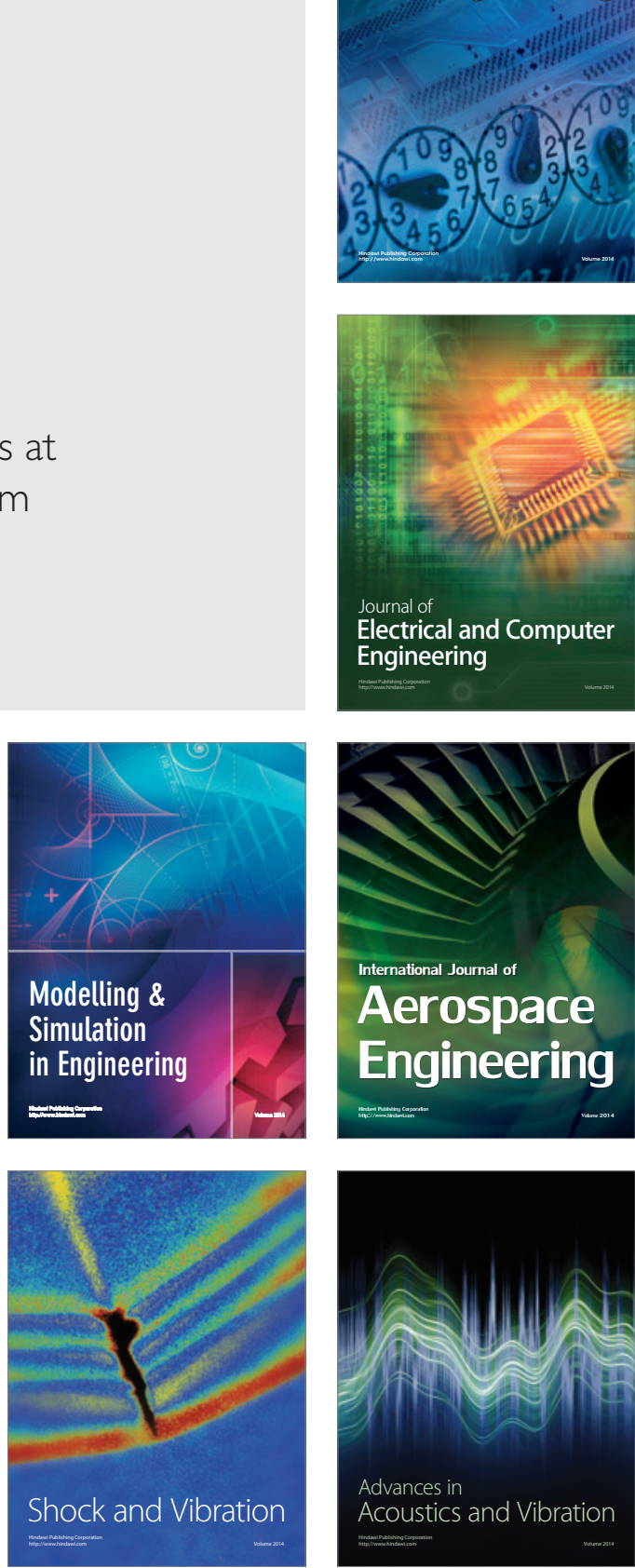